\title{
Application of Modified Mosher's Method for Primary Alcohols with a Methyl Group at C2 Position
}

\author{
Masashi Tsuda, Yuki Toriyabe, Tetsuya Endo, and Jun'ichi Kobayashi* \\ Graduate School of Pharmaceutical Sciences, Hokkaido University; Sapporo 060-0812, Japan. \\ Received November 28, 2002; accepted January 10, 2003
}

\begin{abstract}
The absolute configurations of a series of primary alcohols possessing a branched methyl group at $\mathrm{C} 2$ position were examined on the basis of a modified Mosher's method proposed by Minale et al. It was revealed that in many cases the absolute configurations at $\mathrm{C} 2$ were assignable from the chemical shift differences of geminal protons of the methylene attached to the ester linkage in the $\alpha$-methoxy- $\alpha$-trifluoromethylphenylacetyl (MTPA) derivatives. The scope and limitation of this method are described.
\end{abstract}

Key words absolute configuration; modified Mosher's method; branched primary alcohols

Modified Mosher's method using $\alpha$-methoxy- $\alpha$-trifluoromethylphenylacetyl (MTPA) esters has been recognized as a useful and reliable method for determination of absolute configurations of secondary alcohols and primary amines. ${ }^{1-3)}$ The absolute configurations at such methine carbons could be determined by the regular arrangement: positive and negative chemical shift differences $\left[\Delta \delta\left(\delta_{S}-\delta_{R}\right)\right]$ of the protons can be found on the right and left sides of the MTPA plane, respectively. Nevertheless, it is difficult to assign the absolute configurations of tertiary and primary alcohols since of the irregular arrangements of the $\Delta \delta$ values. ${ }^{1,4)}$ Although attempts to assign the absolute stereochemistry of C2-chiral primary alcohols using its MTPA esters have been demonstrated, there is no conventional method applicable for a limited amount of natural products. ${ }^{5)}$

Minale and coworkers have applied a Mosher's method for determination of absolute stereochemistry of a methyl group at $\mathrm{C} 25$ of steroids with a primary hydroxyl group at $\mathrm{C} 26,{ }^{6,7)}$ and reported that the proton signals of the methylene (C26) attached to the ester linkage in the MTPA derivatives showed a unique split pattern as follows. In the ${ }^{1} \mathrm{H}-\mathrm{NMR}$ spectra of the $(+)-(R)$-MTPA esters, two 26-methylene protons of the $25 S$ isomer were much closer [ $\left.\Delta \delta\left(\delta_{\text {low }}-\delta_{\text {high }}\right) c a .0 .04\right]$ to each other than those $(\Delta \delta \mathrm{ca}$. 0.14$)$ of the $25 R$ isomer, whereas in the $(-)-(S)$-MTPA esters, the mutual relation was reverse. During our studies on stereochemistry of marine natural products, ${ }^{8-12)}$ we have applied this method for primary alcohols with a branched methyl at $\mathrm{C} 2$, and proposed the ab- solute configurations at chiral centers attached to the methyl group. For example, the absolute configuration at C14 of amphidinolide T1 was elucidated on the basis of chemical shift differences and signal patterns of the geminal protons at $\mathrm{C} 13$ of $(S)$ - and $(R)$-MTPA esters of the C13-C21 segment ${ }^{10)}$ (Fig. 1). The methylene protons of $\mathrm{C} 13$ for the $(R)$-MTPA ester were observed as separated double doublet signals at $\delta_{\mathrm{H}}$ 4.03 and $4.26(\Delta \delta 0.23)$, while those for the $(S)$-MTPA ester appeared at $\delta_{\mathrm{H}} 4.09$ and $4.12(\Delta \delta 0.03)$, indicating that absolute configuration at $\mathrm{C} 14$ was $R$. However, this methodology has not been substantiated by examination of compounds with known stereochemistry. Recently, we have applied this method for a series of primary alcohols with a C2-branched methyl whose stereochemistry is known. In this paper we describe the scope and limitation of this methodology on the basis of the present results.

\section{Results and Discussion}

A series of primary alcohols $(\mathbf{1}-\mathbf{1 4})$ with a methyl group (Chart 1), whose absolute configurations are known, were synthesized or obtained commercially, and they were converted into the corresponding $(S)$ - and $(R)$-MTPA esters. Chemical shifts of the oxymethylene protons in the $(S)$ - and $(R)$-MTPA esters of each alcohol in $\mathrm{CDCl}_{3}$ were shown in Table 1 , in which $\Delta \delta$ values denoted chemical shift differences between lower-field protons $\left(\delta_{\text {low }}\right)$ and higher-field ones $\left(\delta_{\text {high }}\right)$ of the oxymethylenes. The $(S)$-MTPA esters of compounds $\mathbf{1}-\mathbf{5}$ possessing $2 S$-configuration had relatively

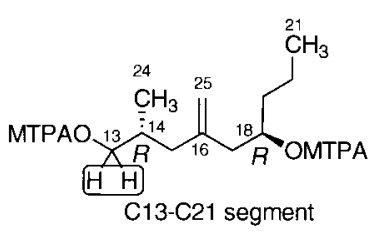

(S)-MTPA

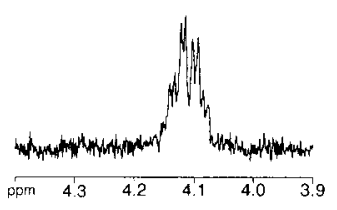

(R)-MTPA

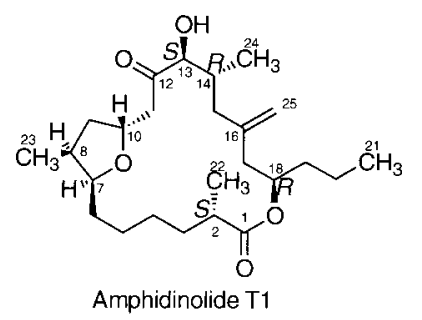

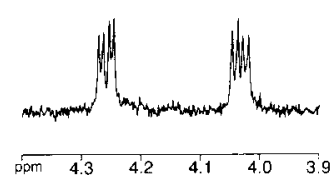

Fig. 1. Signal Patterns of Methylene Protons at C13 of Bis-(S)- and Bis-(R)-MTPA Esters of C13-C21 Segment of Amphidinolide T1 


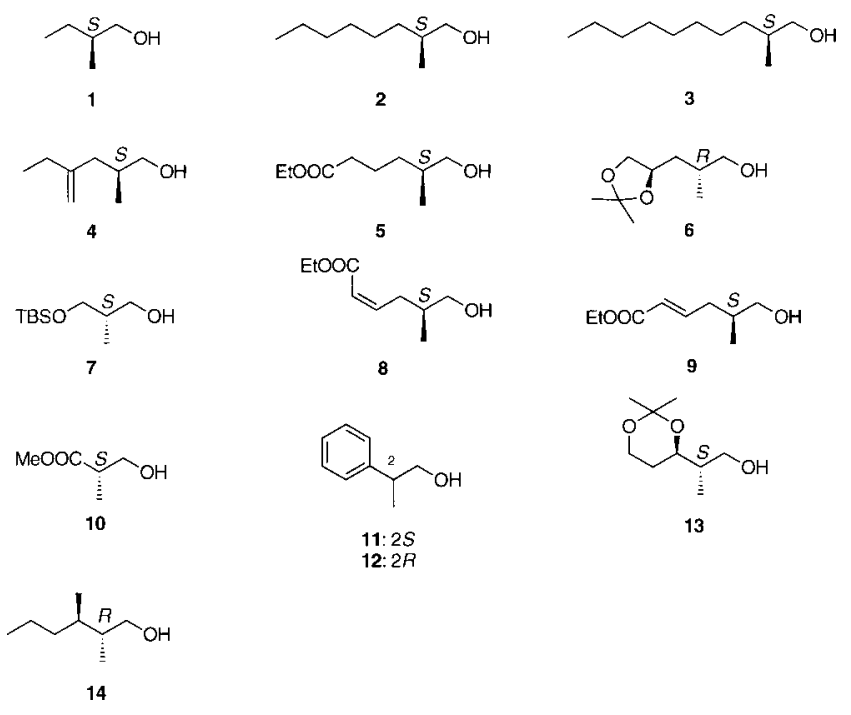

Chart 1

Table 1. Chemical Shifts and $\Delta \delta$ Values of Oxymethylene Protons of MTPA Esters of Primary Alcohols with a Methyl Group at C2-Position (114)

\begin{tabular}{|c|c|c|c|c|}
\hline Compd. & МТPA & $\delta_{\text {low }}^{a)}$ & $\delta_{\text {high }}^{a)}$ & $\Delta \delta^{b)}$ \\
\hline \multirow[t]{2}{*}{1} & $S$ & 4.24 & 4.09 & 0.15 \\
\hline & $R$ & $4.17^{c)}$ & & 0.00 \\
\hline \multirow[t]{2}{*}{2} & $S$ & 4.25 & 4.10 & 0.15 \\
\hline & $R$ & $4.16^{c)}$ & & 0.00 \\
\hline \multirow[t]{2}{*}{3} & $S$ & 4.24 & 4.10 & 0.14 \\
\hline & $R$ & $4.17^{c)}$ & & 0.00 \\
\hline \multirow[t]{2}{*}{4} & $S$ & 4.26 & 4.06 & 0.20 \\
\hline & $R$ & $4.17^{c)}$ & & 0.00 \\
\hline \multirow[t]{2}{*}{5} & $S$ & 4.23 & 4.10 & 0.14 \\
\hline & $R$ & 4.15 & 4.10 & 0.05 \\
\hline \multirow[t]{2}{*}{6} & $S$ & $4.22^{c)}$ & & 0.00 \\
\hline & $R$ & 4.28 & 4.15 & 0.13 \\
\hline \multirow[t]{2}{*}{7} & $S$ & 4.18 & & 0.00 \\
\hline & $R$ & 4.24 & 4.09 & 0.15 \\
\hline \multirow[t]{2}{*}{8} & $S$ & 4.28 & 4.11 & 0.17 \\
\hline & $R$ & $4.21^{c)}$ & & 0.00 \\
\hline \multirow[t]{2}{*}{9} & $S$ & 4.22 & 4.12 & 0.10 \\
\hline & $R$ & 4.22 & 4.11 & 0.11 \\
\hline \multirow[t]{2}{*}{10} & $S$ & 4.44 & 4.36 & 0.08 \\
\hline & $R$ & 4.46 & 4.35 & 0.11 \\
\hline \multirow[t]{2}{*}{11} & $S$ & 4.52 & 4.32 & 0.20 \\
\hline & $R$ & 4.49 & 4.34 & 0.15 \\
\hline \multirow[t]{2}{*}{12} & $S$ & 4.49 & 4.34 & 0.15 \\
\hline & $R$ & 4.52 & 4.32 & 0.20 \\
\hline \multirow[t]{2}{*}{13} & $S$ & 4.38 & 4.32 & 0.06 \\
\hline & $R$ & 4.41 & 4.32 & 0.09 \\
\hline \multirow[t]{2}{*}{14} & $S$ & 4.27 & 4.17 & 0.10 \\
\hline & $R$ & 4.35 & 4.09 & 0.26 \\
\hline
\end{tabular}

a) In $\mathrm{CDCl}_{3}$. b) $\delta_{\text {low }}-\delta_{\text {high }}$ c) $2 \mathrm{H}$ equivalent.

larger $\Delta \delta$ values $(0.20-0.14)$ rather than those $(0-0.05)$ of the $(R)$-MTPA esters. The typical signal pattern of oxymethylene protons was shown in Fig. 2 . The $\Delta \delta$ values observed for the $(S)$ - and $(R)$-MTPA esters of 6 with $2 R$-configuration were 0 and 0.13 , respectively. These results corresponded well to the Minale's proposal. ${ }^{6,7)}$ On the other hand, the $(S)$ and $(R)$-MTPA esters of compound 7 possessing $2 S$-configuration showed opposite pattern: $\Delta \delta_{S}(0)$ was smaller than $\Delta \delta_{R}(0.15)$. Neverthless, the absolute configuration of 7 was
(S)-MTPA $(\Delta \delta=0.20) \quad(R)$-MTPA $(\Delta \delta=0)$

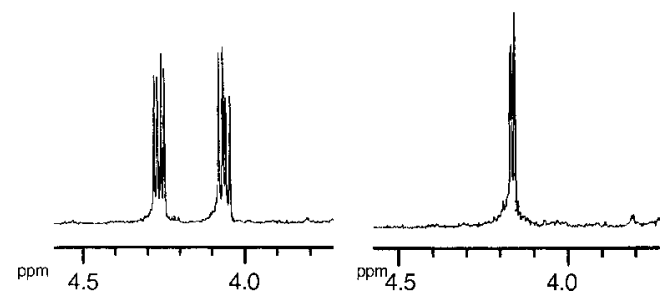

Fig. 2. Signal Patterns of Methylene Protons at $\mathrm{C} 1$ of $(S)$ - and $(R)$-MTPA Esters of Primary Alcohol 4

different from other primary alcohols due to the order of priority between silicon and oxygen atoms.

For compounds 8-12 possessing an unsaturated group such as carbonyl, conjugated ester, or phenyl group, the differences between $\Delta \delta_{S}$ and $\Delta \delta_{R}$ were very small except for that of 8. These observations might result from deshielding effects by both of the phenyl group of MTPA esters and the unsaturated moiety. In the cases of the $(S)$ - and $(R)$-MTPA esters of compounds $\mathbf{1 3}$ and $\mathbf{1 4}$ possessing two consecutive chiral centers at $\mathrm{C} 2$ and $\mathrm{C} 3$, both $\Delta \delta_{S}$ and $\Delta \delta_{R}$ values were relatively large. Furthermore, the difference between $\Delta \delta_{S}$ and $\Delta \delta_{R}$ in 13 was fairly small $(0.03 \mathrm{ppm})$. These phenomena were supposed to come from interfere of free-rotation around the $\mathrm{C} 2-\mathrm{C} 3$ bond, since the methylene protons at $\mathrm{C} 1$ of $\mathbf{1 3}$ showed a split pattern $(\delta 3.25$ and 3.20).

In the present study, we examined the relation between the absolute configuration and oxymethylene proton chemical shifts in the MTPA esters of simple primary alcohols (1-14) with a $\mathrm{C} 2$-branched methyl. Like the case of compounds $1-$ 7 , if the differences between $\Delta \delta_{S}$ and $\Delta \delta_{R}$ are relatively large $(>0.10 \mathrm{ppm})$ they allow the assignment of the absolute configurations at the oxymethine carbons at $\mathrm{C} 2$. On the other hand, like the case of compounds 8-14, if small differences $(<0.05 \mathrm{ppm})$ between $\Delta \delta_{S}$ and $\Delta \delta_{R}$ are observed, it may be difficult to predict the absolute configurations by this method. Particularly, this method could not be applied for C2-branched primary alcohols with an conjugated group or a consecutive chiral center at C3 like compounds $\mathbf{1 3}$ and 14.

\section{Experimental}

General Methods ${ }^{1} \mathrm{H}-\mathrm{NMR}$ spectra were recorded on a Bruker ARX500 spectrometer. FAB mass spectrum was obtained on a JEOL HX-110 spectrometer using nitrobenzyl alcohol as a matrix.

Preparation of MTPA Esters To a $\mathrm{CH}_{2} \mathrm{Cl}_{2}$ solution $(500 \mu \mathrm{l})$ of alcohols $(1 \mathrm{mg})$ was added 4-dimethylaminopyridine $(0.05 \mathrm{mg})$, triethylamine $(50 \mu \mathrm{l})$, and $(R)-(-)-$ or $(S)-(+)-\mathrm{MTPACl}(30 \mu \mathrm{l})$ at room temperature, and stirring was continued for $6 \mathrm{~h}$. $N, N$-Dimethyl-1,3-propanediamine $(30 \mu \mathrm{l})$ was added, and the reaction mixture was stirred for $10 \mathrm{~min}$. After addition of phosphate buffer, the reaction mixture was evaporated in vacuo. The residue was subjected to a $\mathrm{SiO}_{2}$ column with hexane/acetone solvent system to afford an MTPA ester.

$(S)$-MTPA Ester of 1: FAB-MS [M+H] ${ }^{+} m / z$ 305; HR-FAB-MS [M+H] ${ }^{+}$ $m / z 305.1360$ (Calcd for $\left.\mathrm{C}_{15} \mathrm{H}_{20} \mathrm{~F}_{3} \mathrm{O}_{3}: 305.1364\right)$; ${ }^{1} \mathrm{H}-\mathrm{NMR}\left(\mathrm{CDCl}_{3}\right) \delta: 0.909$ $(3 \mathrm{H}, \mathrm{t}, J=7.4 \mathrm{~Hz}), 0.913(3 \mathrm{H}, \mathrm{d}, J=6.8 \mathrm{~Hz}), 1.20(1 \mathrm{H}, \mathrm{m}), 1.41(1 \mathrm{H}, \mathrm{m})$, $1.76(1 \mathrm{H}, \mathrm{m}), 3.55(3 \mathrm{H}, \mathrm{s}), 4.09(1 \mathrm{H}, \mathrm{dd}, J=6.7,10.7 \mathrm{~Hz}), 4.24(1 \mathrm{H}, \mathrm{dd}$, $J=5.7,10.7 \mathrm{~Hz}), 7.39(3 \mathrm{H}, \mathrm{m})$, and $7.52(2 \mathrm{H}, \mathrm{m})$.

(R)-MTPA Ester of 1: FAB-MS [M+H] ${ }^{+} m / z$ 305; HR-FAB-MS [M+H] ${ }^{+}$ $m / z 305.1372$ (Calcd for $\left.\mathrm{C}_{15} \mathrm{H}_{20} \mathrm{~F}_{3} \mathrm{O}_{3}: 305.1364\right) ;{ }^{1} \mathrm{H}-\mathrm{NMR}\left(\mathrm{CDCl}_{3}\right) \delta: 0.89$ $(3 \mathrm{H}, \mathrm{t}, J=7.4 \mathrm{~Hz}), 0.91(3 \mathrm{H}, \mathrm{d}, J=6.8 \mathrm{~Hz}), 1.20(1 \mathrm{H}, \mathrm{m}), 1.38(1 \mathrm{H}, \mathrm{m}), 1.77$ $(1 \mathrm{H}, \mathrm{m}), 3.55(3 \mathrm{H}, \mathrm{s}), 4.17(2 \mathrm{H}, \mathrm{m}), 7.40(3 \mathrm{H}, \mathrm{m})$, and $7.52(2 \mathrm{H}, \mathrm{m})$.

$(S)$-MTPA Ester of 2: FAB-MS [M+H] ${ }^{+} m / z$ 361; HR-FAB-MS [M+H] ${ }^{+}$ $m / z 361.2002$ (Calcd for $\mathrm{C}_{19} \mathrm{H}_{28} \mathrm{~F}_{3} \mathrm{O}_{3}: 361.1991$ ); ${ }^{1} \mathrm{H}-\mathrm{NMR}\left(\mathrm{CDCl}_{3}\right) \delta: 0.89$ $(3 \mathrm{H}, \mathrm{t}, J=7.4 \mathrm{~Hz}), 0.91(3 \mathrm{H}, \mathrm{d}, J=6.8 \mathrm{~Hz}), 1.30(10 \mathrm{H}, \mathrm{m}), 1.76(1 \mathrm{H}, \mathrm{m})$, 
$3.55(3 \mathrm{H}, \mathrm{s}), 4.10(1 \mathrm{H}, \mathrm{dd}, J=6.7,10.7 \mathrm{~Hz}), 4.25(1 \mathrm{H}, \mathrm{dd}, J=5.7,10.7 \mathrm{~Hz})$, $7.39(3 \mathrm{H}, \mathrm{m})$, and $7.52(2 \mathrm{H}, \mathrm{m})$.

$(R)$-MTPA Ester of 2: FAB-MS [M+H] ${ }^{+} m / z$ 361; HR-FAB-MS [M+H] ${ }^{+}$ $m / z 361.1990$ (Calcd for $\left.\mathrm{C}_{19} \mathrm{H}_{28} \mathrm{~F}_{3} \mathrm{O}_{3}: 361.1991\right) ;{ }^{1} \mathrm{H}-\mathrm{NMR}\left(\mathrm{CDCl}_{3}\right) \delta: 0.89$ $(3 \mathrm{H}, \mathrm{t}, J=7.4 \mathrm{~Hz}), 0.91(3 \mathrm{H}, \mathrm{d}, J=6.8 \mathrm{~Hz}), 1.20(10 \mathrm{H}, \mathrm{m}), 1.77(1 \mathrm{H}, \mathrm{m})$, $3.55(3 \mathrm{H}, \mathrm{s}), 4.16(2 \mathrm{H}, \mathrm{m}), 7.40(3 \mathrm{H}, \mathrm{m})$, and $7.52(2 \mathrm{H}, \mathrm{m})$.

(S)-MTPA Ester of 3: FAB-MS [M+H] ${ }^{+} m / z$ 389; HR-FAB-MS $[\mathrm{M}+\mathrm{H}]^{+}$ $m / z 389.2312$ (Calcd for $\mathrm{C}_{21} \mathrm{H}_{32} \mathrm{~F}_{3} \mathrm{O}_{3}: 389.2303$ ); ${ }^{1} \mathrm{H}-\mathrm{NMR}\left(\mathrm{CDCl}_{3}\right) \delta: 0.89$ $(3 \mathrm{H}, \mathrm{t}, J=7.4 \mathrm{~Hz}), 0.91(3 \mathrm{H}, 3 \mathrm{H}, \mathrm{d}, J=6.8 \mathrm{~Hz}), 1.30(14 \mathrm{H}, \mathrm{m}), 1.76(1 \mathrm{H}, \mathrm{m})$, $3.55(3 \mathrm{H}, \mathrm{s}), 4.10(1 \mathrm{H}, \mathrm{dd}, J=6.7,10.7 \mathrm{~Hz}), 4.24(1 \mathrm{H}, \mathrm{dd}, J=5.7,10.7 \mathrm{~Hz})$, $7.39(3 \mathrm{H}, \mathrm{m})$, and $7.52(2 \mathrm{H}, \mathrm{m})$.

(R)-MTPA Ester of 3: FAB-MS $[\mathrm{M}+\mathrm{H}]^{+} m / z$ 389; HR-FAB-MS $[\mathrm{M}+\mathrm{H}]^{+}$ $m / z 389.2308$ (Calcd for $\mathrm{C}_{21} \mathrm{H}_{32} \mathrm{~F}_{3} \mathrm{O}_{3}: 389.2303$ ); ${ }^{1} \mathrm{H}-\mathrm{NMR}\left(\mathrm{CDCl}_{3}\right) \delta: 0.90$ $(3 \mathrm{H}, \mathrm{t}, J=7.4 \mathrm{~Hz}), 0.91(3 \mathrm{H}, 3 \mathrm{H}, \mathrm{d}, J=6.7 \mathrm{~Hz}), 1.30(14 \mathrm{H}, \mathrm{m}), 1.77(1 \mathrm{H}, \mathrm{m})$, $3.55(3 \mathrm{H}, \mathrm{s}), 4.17(2 \mathrm{H}, \mathrm{m}), 7.40(3 \mathrm{H}, \mathrm{m})$, and $7.52(2 \mathrm{H}, \mathrm{m})$.

$(S)$-MTPA Ester of 4: FAB-MS $[\mathrm{M}+\mathrm{H}]^{+} \mathrm{m} / z$ 345; HR-FAB-MS $[\mathrm{M}+\mathrm{H}]^{+}$ $m / z 345.1665$ (Calcd for $\mathrm{C}_{18} \mathrm{H}_{24} \mathrm{~F}_{3} \mathrm{O}_{3}: 345.1677$ ); ${ }^{1} \mathrm{H}-\mathrm{NMR}\left(\mathrm{CDCl}_{3}\right) \delta: 0.90$ $(3 \mathrm{H}, \mathrm{d}, J=6.7 \mathrm{~Hz}), 1.00(3 \mathrm{H}, \mathrm{t}, J=7.4 \mathrm{~Hz}), 1.88(1 \mathrm{H}, \mathrm{m}), 1.98(2 \mathrm{H}, \mathrm{q}$, $J=7.4 \mathrm{~Hz}), 2.09(2 \mathrm{H}, \mathrm{m}), 3.55(3 \mathrm{H}, \mathrm{s}), 4.06(1 \mathrm{H}, \mathrm{dd}, J=6.3,10.9 \mathrm{~Hz}), 4.26$ $(1 \mathrm{H}, \mathrm{dd}, J=5.1,10.9 \mathrm{~Hz}), 4.69(1 \mathrm{H}, \mathrm{s}), 4.79(1 \mathrm{H}, \mathrm{s}), 7.40(3 \mathrm{H}, \mathrm{m})$, and 7.52 $(2 \mathrm{H}, \mathrm{m})$.

$(R)$-MTPA Ester of 4: FAB-MS $[\mathrm{M}+\mathrm{H}]^{+} \mathrm{m} / z$ 345; HR-FAB-MS $[\mathrm{M}+\mathrm{H}]^{+}$ $m / z 345.1672$ (Calcd for $\left.\mathrm{C}_{18} \mathrm{H}_{24} \mathrm{~F}_{3} \mathrm{O}_{3}: 345.1677\right)$ ); ${ }^{1} \mathrm{H}-\mathrm{NMR}\left(\mathrm{CDCl}_{3}\right) \delta: 0.93$ $(3 \mathrm{H}, \mathrm{d}, J=6.7 \mathrm{~Hz}), 1.00(3 \mathrm{H}, \mathrm{t}, J=7.4 \mathrm{~Hz}), 1.87(1 \mathrm{H}, \mathrm{m}), 1.96(2 \mathrm{H}, \mathrm{q}$, $J=7.4 \mathrm{~Hz}), 2.08(2 \mathrm{H}, \mathrm{m}), 3.55(3 \mathrm{H}, \mathrm{s}), 4.17(2 \mathrm{H}, \mathrm{m}), 4.66(1 \mathrm{H}, \mathrm{s}), 4.78(1 \mathrm{H}$, $\mathrm{s}), 7.40(3 \mathrm{H}, \mathrm{m})$, and $7.52(2 \mathrm{H}, \mathrm{m})$.

$(S)$-MTPA Ester of 5: FAB-MS $[\mathrm{M}+\mathrm{H}]^{+} \mathrm{m} / z$ 391; HR-FAB-MS [M+H $]^{+}$ $m / z 391.1728$ (Calcd for $\mathrm{C}_{19} \mathrm{H}_{26} \mathrm{~F}_{3} \mathrm{O}_{5}: 391.1732$ ); ${ }^{1} \mathrm{H}-\mathrm{NMR}\left(\mathrm{CDCl}_{3}\right) \delta: 0.93$ $(3 \mathrm{H}, \mathrm{d}, J=6.7 \mathrm{~Hz}), 1.25(3 \mathrm{H}, \mathrm{t}, J=7.2 \mathrm{~Hz}), 1.19(1 \mathrm{H}, \mathrm{m}), 1.37(1 \mathrm{H}, \mathrm{m}), 1.59$ $(1 \mathrm{H}, \mathrm{m}), 1.68(1 \mathrm{H}, \mathrm{m}), 1.87(1 \mathrm{H}, \mathrm{m}), 2.25(2 \mathrm{H}, \mathrm{t}, J=7.5 \mathrm{~Hz}), 3.55(3 \mathrm{H}, \mathrm{s})$, $4.10(1 \mathrm{H}, \mathrm{dd}, J=6.0,10.7 \mathrm{~Hz}), 4.11(2 \mathrm{H}, \mathrm{q}, J=7.2 \mathrm{~Hz}), 4.23(1 \mathrm{H}, \mathrm{dd}, J=3.1$, $10.7 \mathrm{~Hz}), 7.40(3 \mathrm{H}, \mathrm{m})$, and $7.52(2 \mathrm{H}, \mathrm{m})$.

$(R)$-MTPA Ester of 5: FAB-MS $[\mathrm{M}+\mathrm{H}]^{+} m / z$ 391; HR-FAB-MS $[\mathrm{M}+\mathrm{H}]^{+}$ $m / z 391.1737$ (Calcd for $\left.\mathrm{C}_{19} \mathrm{H}_{26} \mathrm{~F}_{3} \mathrm{O}_{5}: 391.1732\right)$; ${ }^{1} \mathrm{H}-\mathrm{NMR}\left(\mathrm{CDCl}_{3}\right) \delta: 0.93$ $(3 \mathrm{H}, \mathrm{d}, J=6.8 \mathrm{~Hz}), 1.25(3 \mathrm{H}, \mathrm{t}, J=7.2 \mathrm{~Hz}), 1.17(1 \mathrm{H}, \mathrm{m}), 1.36(1 \mathrm{H}, \mathrm{m}), 1.57$ $(1 \mathrm{H}, \mathrm{m}), 1.69(1 \mathrm{H}, \mathrm{m}), 1.86(1 \mathrm{H}, \mathrm{m}), 2.24(2 \mathrm{H}, \mathrm{dt}, J=2.1,7.0 \mathrm{~Hz}), 3.55(3 \mathrm{H}$, s), $4.10(1 \mathrm{H}, \mathrm{dd}, J=6.3,10.9 \mathrm{~Hz}), 4.14(2 \mathrm{H}, \mathrm{q}, J=7.2 \mathrm{~Hz}), 4.15(1 \mathrm{H}, \mathrm{dd}$, $J=5.1,10.7 \mathrm{~Hz}), 7.40(3 \mathrm{H}, \mathrm{m})$, and $7.47(2 \mathrm{H}, \mathrm{m})$.

$(S)$-MTPA Ester of 6: FAB-MS [M+H] ${ }^{+} m / z$ 391; HR-FAB-MS [M+H] ${ }^{+}$ $m / z 391.1741$ (Calcd for $\mathrm{C}_{19} \mathrm{H}_{26} \mathrm{~F}_{3} \mathrm{O}_{5}: 391.1732$ ); ${ }^{1} \mathrm{H}-\mathrm{NMR}\left(\mathrm{CDCl}_{3}\right) \delta: 0.97$ $(3 \mathrm{H}, \mathrm{d}, J=6.9 \mathrm{~Hz}), 1.33(3 \mathrm{H}, \mathrm{s}), 1.37(3 \mathrm{H}, \mathrm{s}), 1.63(2 \mathrm{H}, \mathrm{m}), 2.09(1 \mathrm{H}, \mathrm{m})$, $3.45(1 \mathrm{H}, \mathrm{t}, J=7.0 \mathrm{~Hz}), 3.55(3 \mathrm{H}, \mathrm{s}), 4.02(1 \mathrm{H}, \mathrm{dd}, J=5.9,7.8 \mathrm{~Hz}), 4.13(1 \mathrm{H}$, $\mathrm{m}), 4.22(2 \mathrm{H}, \mathrm{m}), 7.41(3 \mathrm{H}, \mathrm{m})$, and $7.52(2 \mathrm{H}, \mathrm{m})$.

(R)-MTPA Ester of 6: FAB-MS [M+H] ${ }^{+} m / z$ 391; HR-FAB-MS $[\mathrm{M}+\mathrm{H}]^{+}$ $m / z 391.1729$ (Calcd for $\mathrm{C}_{19} \mathrm{H}_{26} \mathrm{~F}_{3} \mathrm{O}_{5}: 391.1732$ ); ${ }^{1} \mathrm{H}-\mathrm{NMR}\left(\mathrm{CDCl}_{3}\right) \delta: 0.98$ $(3 \mathrm{H}, \mathrm{d}, J=6.9 \mathrm{~Hz}), 1.33(3 \mathrm{H}, \mathrm{s}), 1.37(3 \mathrm{H}, \mathrm{s}), 1.64(1 \mathrm{H}, \mathrm{m}), 2.10(1 \mathrm{H}, \mathrm{m})$, $3.45(1 \mathrm{H}, \mathrm{t}, J=7.5 \mathrm{~Hz}), 3.55(3 \mathrm{H}, \mathrm{s}), 4.03(1 \mathrm{H}, \mathrm{dd}, J=6.0,7.9 \mathrm{~Hz}), 4.14(1 \mathrm{H}$, $\mathrm{m}), 4.15(1 \mathrm{H}, \mathrm{dd}, J=6.3,10.8 \mathrm{~Hz}), 4.28(1 \mathrm{H}, \mathrm{dd}, J=5.7,10.8 \mathrm{~Hz}), 7.40(3 \mathrm{H}$, $\mathrm{m})$, and $7.52(2 \mathrm{H}, \mathrm{m})$.

$(S)$-MTPA Ester of 7: FAB-MS [M+H] ${ }^{+} m / z$ 421; HR-FAB-MS [M+H] ${ }^{+}$ $m / z 421.2016$ (Calcd for $\left.\mathrm{C}_{20} \mathrm{H}_{32} \mathrm{~F}_{3} \mathrm{O}_{4} \mathrm{Si}: 421.2022\right)$; ${ }^{1} \mathrm{H}-\mathrm{NMR}\left(\mathrm{CDCl}_{3}\right) \delta$ : $0.00(6 \mathrm{H}, \mathrm{s}), 0.89(12 \mathrm{H}, \mathrm{m}), 1.96(1 \mathrm{H}, \mathrm{m}), 3.45(2 \mathrm{H}, \mathrm{m}), 3.55(3 \mathrm{H}, \mathrm{s}), 4.18$ $(2 \mathrm{H}, \mathrm{m}), 7.41(3 \mathrm{H}, \mathrm{m})$, and $7.52(2 \mathrm{H}, \mathrm{m})$.

(R)-MTPA Ester of 7: FAB-MS [M+H] ${ }^{+} m / z$ 421; HR-FAB-MS [M+H] ${ }^{+}$ $m / z 421.2026$ (Calcd for $\left.\mathrm{C}_{20} \mathrm{H}_{32} \mathrm{~F}_{3} \mathrm{O}_{4} \mathrm{Si}: 421.2022\right)$; ${ }^{1} \mathrm{H}-\mathrm{NMR}\left(\mathrm{CDCl}_{3}\right) \delta$ : $0.00(6 \mathrm{H}, \mathrm{s}), 0.88(12 \mathrm{H}, \mathrm{m}), 1.95(1 \mathrm{H}, \mathrm{m}), 3.46(2 \mathrm{H}, \mathrm{m}), 3.55(3 \mathrm{H}, \mathrm{s}), 4.09$ $(1 \mathrm{H}, \mathrm{dd}, J=5.9,7.8 \mathrm{~Hz}), 4.24(1 \mathrm{H}, \mathrm{dd}, J=5.7,10.8 \mathrm{~Hz}), 7.41(3 \mathrm{H}, \mathrm{m})$, and $7.52(2 \mathrm{H}, \mathrm{m})$.

$(S)$-MTPA Ester of 8: FAB-MS $[\mathrm{M}+\mathrm{H}]^{+} m / z$ 389; HR-FAB-MS $[\mathrm{M}+\mathrm{H}]^{+}$ $m / z 389.1574$ (Calcd for $\mathrm{C}_{19} \mathrm{H}_{24} \mathrm{~F}_{3} \mathrm{O}_{5}: 389.1576$ ); ${ }^{1} \mathrm{H}-\mathrm{NMR}\left(\mathrm{CDCl}_{3}\right) \delta: 0.96$ $(3 \mathrm{H}, \mathrm{d}, J=6.8 \mathrm{~Hz}), 1.28(3 \mathrm{H}, \mathrm{t}, J=7.0 \mathrm{~Hz}), 2.05(1 \mathrm{H}, \mathrm{m}), 2.65(2 \mathrm{H}, \mathrm{t}$, $J=7.3 \mathrm{~Hz}), 3.55(3 \mathrm{H}, \mathrm{s}), 4.11(1 \mathrm{H}, \mathrm{dd}, J=6.8,10.8 \mathrm{~Hz}), 4.16(2 \mathrm{H}, \mathrm{q}$, $J=7.0 \mathrm{~Hz}), 4.28(1 \mathrm{H}, \mathrm{dd}, J=5.7,10.8 \mathrm{~Hz}), 5.83(1 \mathrm{H}, \mathrm{d}, J=11.6 \mathrm{~Hz}), 6.17$ $(1 \mathrm{H}, \mathrm{m}), 7.40(3 \mathrm{H}, \mathrm{m})$, and $7.51(2 \mathrm{H}, \mathrm{m})$.

$(R)$-MTPA Ester of 8: FAB-MS [M+H] ${ }^{+} m / z 389$; HR-FAB-MS $[\mathrm{M}+\mathrm{H}]^{+}$ $m / z 389.1576$ (Calcd for $\left.\mathrm{C}_{19} \mathrm{H}_{24} \mathrm{~F}_{3} \mathrm{O}_{5}: 389.1576\right)$ ) ${ }^{1} \mathrm{H}-\mathrm{NMR}\left(\mathrm{CDCl}_{3}\right) \delta: 0.97$ $(3 \mathrm{H}, \mathrm{d}, J=6.8 \mathrm{~Hz}), 1.28(3 \mathrm{H}, \mathrm{t}, J=7.0 \mathrm{~Hz}), 2.05(1 \mathrm{H}, \mathrm{m}), 2.65(2 \mathrm{H}, \mathrm{t}$, $J=7.3 \mathrm{~Hz}), 3.55(3 \mathrm{H}, \mathrm{s}), 4.16(2 \mathrm{H}, \mathrm{q}, J=7.0 \mathrm{~Hz}), 4.21(2 \mathrm{H}, \mathrm{m}), 5.82(1 \mathrm{H}, \mathrm{d}$, $J=11.6 \mathrm{~Hz}), 6.16(1 \mathrm{H}, \mathrm{m}), 7.40(3 \mathrm{H}, \mathrm{m})$, and $7.51(2 \mathrm{H}, \mathrm{m})$.

$(S)$-MTPA Ester of 9: FAB-MS [M+H] ${ }^{+} m / z$ 389; HR-FAB-MS [M+H] ${ }^{+}$ $m / z 389.1581$ (Calcd for $\left.\mathrm{C}_{19} \mathrm{H}_{24} \mathrm{~F}_{3} \mathrm{O}_{5}: 389.1576\right) ;{ }^{1} \mathrm{H}-\mathrm{NMR}\left(\mathrm{CDCl}_{3}\right) \delta: 0.96$ $(3 \mathrm{H}, \mathrm{d}, J=6.8 \mathrm{~Hz}), 1.29(3 \mathrm{H}, \mathrm{t}, J=7.0 \mathrm{~Hz}), 2.08(2 \mathrm{H}, \mathrm{m}), 2.27(1 \mathrm{H}, \mathrm{m}), 3.54$ $(3 \mathrm{H}, \mathrm{s}), 4.12(1 \mathrm{H}, \mathrm{dd}, J=5.4,10.8 \mathrm{~Hz}), 4.19(2 \mathrm{H}, \mathrm{q}, J=7.0 \mathrm{~Hz}), 4.22(1 \mathrm{H}, \mathrm{dd}$, $J=5.8,10.8 \mathrm{~Hz}), 5.80(1 \mathrm{H}, \mathrm{d}, J=15.5 \mathrm{~Hz}), 6.87(1 \mathrm{H}, \mathrm{m}), 7.40(3 \mathrm{H}, \mathrm{m})$, and
$7.51(2 \mathrm{H}, \mathrm{m})$.

$(R)$-MTPA Ester of 9: FAB-MS [M+H] ${ }^{+} m / z$ 389; HR-FAB-MS [M+H] ${ }^{+}$ $m / z 389.1579$ (Calcd for $\left.\mathrm{C}_{19} \mathrm{H}_{24} \mathrm{~F}_{3} \mathrm{O}_{5}: 389.1576\right) ;{ }^{1} \mathrm{H}-\mathrm{NMR}\left(\mathrm{CDCl}_{3}\right) \delta: 0.97$ $(3 \mathrm{H}, \mathrm{d}, J=6.8 \mathrm{~Hz}), 1.29(3 \mathrm{H}, \mathrm{t}, J=7.0 \mathrm{~Hz}), 2.12(2 \mathrm{H}, \mathrm{m}), 2.24(1 \mathrm{H}, \mathrm{m}), 3.54$ $(3 \mathrm{H}, \mathrm{s}), 4.11(1 \mathrm{H}, \mathrm{dd}, J=5.7,10.9 \mathrm{~Hz}), 4.18(2 \mathrm{H}, \mathrm{q}, J=7.0 \mathrm{~Hz}), 4.22(1 \mathrm{H}, \mathrm{dd}$, $J=5.9,10.9 \mathrm{~Hz}), 5.78(1 \mathrm{H}, \mathrm{d}, J=15.5 \mathrm{~Hz}), 6.85(1 \mathrm{H}, \mathrm{m}), 7.41(3 \mathrm{H}, \mathrm{m})$, and $7.51(2 \mathrm{H}, \mathrm{m})$.

(S)-MTPA Ester of 10: FAB-MS $\left[\mathrm{M}+\mathrm{H}^{+} \mathrm{m} / \mathrm{z}\right.$ 335; HR-FAB-MS $[\mathrm{M}+\mathrm{H}]^{+} \quad m / z 335.1114$ (Calcd for $\mathrm{C}_{15} \mathrm{H}_{18} \mathrm{~F}_{3} \mathrm{O}_{5}: 335.1106$ ); ${ }^{1} \mathrm{H}-\mathrm{NMR}$ $\left(\mathrm{CDCl}_{3}\right) \delta: 1.24(3 \mathrm{H}, \mathrm{d}, J=6.8 \mathrm{~Hz}), 2.58(1 \mathrm{H}, \mathrm{m}), 3.55(3 \mathrm{H}, \mathrm{s}), 3.67(3 \mathrm{H}, \mathrm{s})$, $4.36(1 \mathrm{H}, \mathrm{dd}, J=5.4,10.8 \mathrm{~Hz}), 4.44(1 \mathrm{H}, \mathrm{dd}, J=5.8,10.8 \mathrm{~Hz}), 7.41(3 \mathrm{H}, \mathrm{m})$, and $7.51(2 \mathrm{H}, \mathrm{m})$.

$(R)$-MTPA Ester of 10: FAB-MS $[\mathrm{M}+\mathrm{H}]^{+} m / z$ 335; HR-FAB-MS $[\mathrm{M}+\mathrm{H}]^{+} \mathrm{m} / \mathrm{z} 335.1101$ (Calcd for $\mathrm{C}_{15} \mathrm{H}_{18} \mathrm{~F}_{3} \mathrm{O}_{5}:$ 335.1106); ${ }^{1} \mathrm{H}-\mathrm{NMR}$ $\left(\mathrm{CDCl}_{3}\right) \delta: 1.25(3 \mathrm{H}, \mathrm{d}, J=6.9 \mathrm{~Hz}), 2.59(1 \mathrm{H}, \mathrm{m}), 3.55(3 \mathrm{H}, \mathrm{s}), 3.67(3 \mathrm{H}, \mathrm{s})$, $4.35(1 \mathrm{H}, \mathrm{dd}, J=5.3,10.9 \mathrm{~Hz}), 4.46(1 \mathrm{H}, \mathrm{dd}, J=5.8,10.9 \mathrm{~Hz}), 7.41(3 \mathrm{H}, \mathrm{m})$, and $7.51(2 \mathrm{H}, \mathrm{m})$.

$(S)$-MTPA Ester of 11: FAB-MS $[\mathrm{M}+\mathrm{H}]^{+} \mathrm{m} / \mathrm{z}$ 353; HR-FAB-MS $[\mathrm{M}+\mathrm{H}]^{+} \quad m / z \quad 353.1370$ (Calcd for $\mathrm{C}_{19} \mathrm{H}_{20} \mathrm{~F}_{3} \mathrm{O}_{3}: 353.1364$ ); ${ }^{1} \mathrm{H}-\mathrm{NMR}$ $\left(\mathrm{CDCl}_{3}\right) \delta: 1.30(2 \mathrm{H}, \mathrm{d}, J=6.9 \mathrm{~Hz}), 3.19(1 \mathrm{H}, \mathrm{m}), 3.43(3 \mathrm{H}, \mathrm{s}), 4.32(1 \mathrm{H}, \mathrm{dd}$, $J=7.0,10.8 \mathrm{~Hz}), 4.52(1 \mathrm{H}, \mathrm{dd}, J=6.7,10.8 \mathrm{~Hz})$, and $7.20-7.42(10 \mathrm{H}, \mathrm{m})$.

(R)-MTPA Ester of 11: FAB-MS $[\mathrm{M}+\mathrm{H}]^{+} \mathrm{m} / \mathrm{z}$ 353; HR-FAB-MS $[\mathrm{M}+\mathrm{H}]^{+} \mathrm{m} / \mathrm{z} 353.1360$ (Calcd for $\mathrm{C}_{19} \mathrm{H}_{20} \mathrm{~F}_{3} \mathrm{O}_{3}:$ 353.1364); ${ }^{1} \mathrm{H}-\mathrm{NMR}$ $\left(\mathrm{CDCl}_{3}\right) \delta: 1.41(2 \mathrm{H}, \mathrm{d}, J=6.9 \mathrm{~Hz}), 3.17(1 \mathrm{H}, \mathrm{m}), 3.40(3 \mathrm{H}, \mathrm{s}), 4.34(1 \mathrm{H}, \mathrm{dd}$, $J=6.8,11.0 \mathrm{~Hz}), 4.49(1 \mathrm{H}, \mathrm{dd}, J=7.5,11.0 \mathrm{~Hz})$, and $7.19-7.39(10 \mathrm{H}, \mathrm{m})$.

$(S)$-MTPA Ester of 12: FAB-MS $[\mathrm{M}+\mathrm{H}]^{+} \mathrm{m} / \mathrm{z}$ 353; HR-FAB-MS $[\mathrm{M}+\mathrm{H}]^{+} \mathrm{m} / \mathrm{z} 353.1362$ (Calcd for $\mathrm{C}_{19} \mathrm{H}_{20} \mathrm{~F}_{3} \mathrm{O}_{3}: 353.1364$ ); ${ }^{1} \mathrm{H}-\mathrm{NMR}$ $\left(\mathrm{CDCl}_{3}\right) \delta: 1.41(2 \mathrm{H}, \mathrm{d}, J=6.9 \mathrm{~Hz}), 3.17(1 \mathrm{H}, \mathrm{m}), 3.40(3 \mathrm{H}, \mathrm{s}), 4.34(1 \mathrm{H}, \mathrm{dd}$, $J=6.8,11.0 \mathrm{~Hz}), 4.49(1 \mathrm{H}, \mathrm{dd}, J=7.5,11.0 \mathrm{~Hz})$, and $7.19-7.39(10 \mathrm{H}, \mathrm{m})$.

(R)-MTPA Ester of 12: FAB-MS [M+H] ${ }^{+} \mathrm{m} / \mathrm{z}$ 353; HR-FAB-MS $[\mathrm{M}+\mathrm{H}]^{+} \quad m / z \quad 353.1366$ (Calcd for $\mathrm{C}_{19} \mathrm{H}_{20} \mathrm{~F}_{3} \mathrm{O}_{3}: 353.1364$ ); ${ }^{1} \mathrm{H}-\mathrm{NMR}$ $\left(\mathrm{CDCl}_{3}\right) \delta: 1.30(2 \mathrm{H}, \mathrm{d}, J=6.9 \mathrm{~Hz}), 3.19(1 \mathrm{H}, \mathrm{m}), 3.43(3 \mathrm{H}, \mathrm{s}), 4.32(1 \mathrm{H}, \mathrm{dd}$, $J=7.0,10.8 \mathrm{~Hz}), 4.52(1 \mathrm{H}, \mathrm{dd}, J=6.7,10.8 \mathrm{~Hz})$, and $7.20-7.42(10 \mathrm{H}, \mathrm{m})$.

$(S)$-MTPA Ester of 13: FAB-MS $[\mathrm{M}+\mathrm{H}]^{+} \mathrm{m} / \mathrm{z}$ 391; HR-FAB-MS $[\mathrm{M}+\mathrm{H}]^{+} \quad m / z \quad 391.1736$ (Calcd for $\mathrm{C}_{19} \mathrm{H}_{26} \mathrm{~F}_{3} \mathrm{O}_{5}: 391.1732$ ); ${ }^{1} \mathrm{H}-\mathrm{NMR}$ $\left(\mathrm{CDCl}_{3}\right) \delta: 0.92(3 \mathrm{H}, \mathrm{d}, J=7.1 \mathrm{~Hz}), 1.30(3 \mathrm{H}, \mathrm{s}), 1.34(3 \mathrm{H}, \mathrm{s}), 1.45(1 \mathrm{H}, \mathrm{m})$, $1.55(1 \mathrm{H}, \mathrm{m}), 1.87(1 \mathrm{H}, \mathrm{m}), 3.55(3 \mathrm{H}, \mathrm{s}), 3.66(1 \mathrm{H}, \mathrm{m}), 3.83(1 \mathrm{H}, \mathrm{m}), 3.89$ $(1 \mathrm{H}, \mathrm{m}), 4.32(1 \mathrm{H}, \mathrm{dd}, J=3.9,10.7 \mathrm{~Hz}), 4.38(1 \mathrm{H}, \mathrm{dd}, J=5.5,10.7 \mathrm{~Hz}), 7.40$ $(3 \mathrm{H}, \mathrm{m})$, and $7.52(2 \mathrm{H}, \mathrm{m})$.

(R)-MTPA Ester of 13: FAB-MS $[\mathrm{M}+\mathrm{H}]^{+} m / z$ 391; HR-FAB-MS $[\mathrm{M}+\mathrm{H}]^{+} \quad m / z \quad 391.1735$ (Calcd for $\mathrm{C}_{19} \mathrm{H}_{26} \mathrm{~F}_{3} \mathrm{O}_{5}:$ 391.1732); ${ }^{1} \mathrm{H}-\mathrm{NMR}$ $\left(\mathrm{CDCl}_{3}\right) \delta: 0.90(3 \mathrm{H}, \mathrm{d}, J=6.9 \mathrm{~Hz}), 1.36(6 \mathrm{H}, \mathrm{s}), 1.46(1 \mathrm{H}, \mathrm{m}), 1.55(1 \mathrm{H}, \mathrm{m})$, $1.89(1 \mathrm{H}, \mathrm{m}), 3.55(3 \mathrm{H}, \mathrm{s}), 3.69(1 \mathrm{H}, \mathrm{m}), 3.85(1 \mathrm{H}, \mathrm{m}), 3.90(1 \mathrm{H}, \mathrm{m}), 4.32$ $(1 \mathrm{H}, \mathrm{dd}, J=3.9,10.7 \mathrm{~Hz}), 4.41(1 \mathrm{H}, \mathrm{dd}, J=5.5,10.7 \mathrm{~Hz}), 7.40(3 \mathrm{H}, \mathrm{m})$, and $7.52(2 \mathrm{H}, \mathrm{m})$.

(S)-MTPA Ester of 14: FAB-MS $[\mathrm{M}+\mathrm{H}]^{+} \mathrm{m} / \mathrm{z}$ 347; HR-FAB-MS $[\mathrm{M}+\mathrm{H}]^{+} \mathrm{m} / \mathrm{z} 347.1829$ (Calcd for $\mathrm{C}_{18} \mathrm{H}_{26} \mathrm{~F}_{3} \mathrm{O}_{3}: 347.1834$ ); ${ }^{1} \mathrm{H}-\mathrm{NMR}$ $\left(\mathrm{CDCl}_{3}\right) \delta: 0.84(9 \mathrm{H}, \mathrm{m}), 1.07(1 \mathrm{H}, \mathrm{m}), 1.18(1 \mathrm{H}, \mathrm{m}), 1.27(1 \mathrm{H}, \mathrm{m}), 1.36$ $(1 \mathrm{H}, \mathrm{m}), 1.47(1 \mathrm{H}, \mathrm{m}), 1.80(1 \mathrm{H}, \mathrm{m}), 3.55(3 \mathrm{H}, \mathrm{s}), 4.17(1 \mathrm{H}, \mathrm{dd}, J=7.5$, $10.8 \mathrm{~Hz}), 4.27(1 \mathrm{H}, \mathrm{dd}, J=5.6,10.8 \mathrm{~Hz}), 7.40(3 \mathrm{H}, \mathrm{m})$, and $7.52(3 \mathrm{H}, \mathrm{m})$.

(R)-MTPA Ester of 14: FAB-MS [M+H] ${ }^{+} \mathrm{m} / z$ 347; HR-FAB-MS $[\mathrm{M}+\mathrm{H}]^{+} \quad m / z \quad 347.1838$ (Calcd for $\mathrm{C}_{18} \mathrm{H}_{26} \mathrm{~F}_{3} \mathrm{O}_{3}: 347.1834$ ); ${ }^{1} \mathrm{H}-\mathrm{NMR}$ $\left(\mathrm{CDCl}_{3}\right) \delta: 0.83(9 \mathrm{H}, \mathrm{m}), 1.08(1 \mathrm{H}, \mathrm{m}), 1.18(1 \mathrm{H}, \mathrm{m}), 1.28(1 \mathrm{H}, \mathrm{m}), 1.35$ $(1 \mathrm{H}, \mathrm{m}), 1.48(1 \mathrm{H}, \mathrm{m}), 1.80(1 \mathrm{H}, \mathrm{m}), 3.55(3 \mathrm{H}, \mathrm{s}), 4.09(1 \mathrm{H}, \mathrm{dd}, J=7.6$, $10.8 \mathrm{~Hz}), 4.35(1 \mathrm{H}, \mathrm{dd}, J=5.6,10.8 \mathrm{~Hz}), 7.40(3 \mathrm{H}, \mathrm{m})$, and $7.52(3 \mathrm{H}, \mathrm{m})$.

Acknowledgments This work was partly supported by a Grant-in-Aid for Scientific Research from the Ministry of Education, Culture, Sports, Science, and Technology of Japan.

\section{References}

1) Ohtani I., Kusumi T., Kashman Y., Kakisawa H., J. Am. Chem. Soc., 113, 4092-4095 (1991).

2) Kusumi T., Hamada T., Ishitsuka M. O., Ohtani I., Kakisawa H., J. Org. Chem., 57, 1033-1035 (1992).

3) Kusumi T., Fukushima T., Ohtani I., Kakisawa H., Tetrahedron Lett., 32, 2939-2942 (1991).

4) Latypov S. K., Ferreiro M. J., Quiñoá E., Riguera R., J. Am. Chem. Soc., 120, 4741-4751 (1998).

5) Yasuhara F., Yamaguchi S., Tetrahedron Lett., 47, 4085-4088 (1977).

6) De Riccardis F., Minale L., Riccio R., Giovannitti B., Iorizzi M., Debitus C., Gazz. Chim. Ital., 123, 79-86 (1993).

7) Finamore E., Minale L., Riccio R., Rinaldo G., Zollo F., J. Org. Chem., 56, 1146-1153 (1991). 
8) Fontana A., Ishibashi M., Kobayashi J., Tetrahedron, 54, 2041-2048 (1998).

9) Shigemori H., Sato Y., Kagata T., Kobayashi J., J. Nat. Prod., 62, 372-374 (1999).

10) Tsuda M., Endo T., Kobayashi J., J. Org. Chem., 65, 1349-1352 (2000).
11) Kobayashi J., Kubota T., Endo T., Tsuda M., J. Org. Chem., 66, 134 142 (2001).

12) Kubota T., Tsuda M., Kobayashi J., Org. Lett., 3, 1363-1366 (2001).

13) Kubota T., Tsuda M., Kobayashi J., J. Org. Chem., 67, 1651-1656 (2002). 